\title{
Association of serotonin receptor 2a haplotypes with obsessive-compulsive disorder and its treatment response in Iranian patients: a genetic and pharmacogenetic study
}

This article was published in the following Dove Press journal:

Neuropsychiatric Disease and Treatment

\author{
Marzie Sina' \\ Abolhassan Ahmadiani' \\ Sareh Asadi² \\ Jamal Shams ${ }^{3}$ \\ 'Neuroscience Research Center, \\ Shahid Beheshti University of Medical \\ Sciences, Tehran, Iran; ${ }^{2}$ NeuroBiology \\ Research Center, Shahid Beheshti \\ University of Medical Sciences, Tehran, \\ Iran; ${ }^{3}$ Behavioral Sciences Research \\ Center, Shahid Beheshti University \\ of Medical Sciences, Tehran, Iran
}

Correspondence: Sareh Asadi NeuroBiology Research Center, Shahid Beheshti University of Medical Sciences, Daneshjoo Blvd, PO Box 19615-II78, Tehran, Iran

Tel +98 2I 22429768

Fax +98 212 2432047

Email s.asadi@sbmu.ac.ir

Jamal Shams

Behavioral Sciences Research Center, Imam Hossain Educational Hospital,

Shahid Beheshti University of Medical

Sciences, Madani Avenue, PO Box

I6|7763|4I, Tehran, Iran

Tel/fax +98217 7553074

Emailjsshams@sbmu.ac.ir
Introduction: Obsessive-compulsive disorder (OCD) is a debilitating psychiatric disorder causing intrusive thoughts or repetitive behaviors. Serotonin reuptake inhibitors are used for OCD treatment, but $40 \%-60 \%$ of patients do not respond to them adequately. In this study, the associations of serotonin receptor 2a polymorphisms rs6311 and rs6313 with OCD, its familial form and fluvoxamine treatment response in Iranian population were investigated.

Patients and methods: Association analyses were conducted in 293 OCD cases fulfilling the Diagnostic and Statistical Manual of Mental Disorders (DSM)-IV-TR and 245 controls. Pharmacotherapy was defined as 12 weeks of treatment with fluvoxamine (150-300 mg). Treatment response was considered as $>25 \%$ reduction in Yale-Brown Obsessive Compulsive Scale score. Genotyping was performed by means of PCR-RFLP.

Results: The results showed no association of rs6311 or rs6313 with OCD, but their haplotypes had different distribution patterns in cases and controls. Moreover, rs6313 was associated with the familial form of OCD in females significantly $(P=0.005)$ under the recessive genetic model. Moreover, rs6311-rs6313 haplotypes were associated with fluvoxamine treatment response in OCD patients with more AC and less AT in responders.

Conclusion: HTR2A haplotypes are associated with OCD and its treatment response with a fluvoxamine in Iranian patients. Furthermore, the observed association of rs6313 with the familial form of OCD in females suggests different genetic background of OCD familial and non-familial forms, which needs further investigation.

Keywords: family history, fluvoxamine, treatment response, rs6311, rs6313

\section{Introduction}

Obsessive-compulsive disorder (OCD) is a chronic debilitating disorder characterized by recurring unwelcome and intrusive thoughts, beliefs, impulses, images or urges (obsession) that typically cause anxiety, accompanied by repetitive mental or behavioral acts (compulsion) that individuals iterate to relieve their tension. OCD sufferers often realize that their obsessions and compulsions are irrational and excessive; nevertheless, they are not able to stop them. ${ }^{1,2}$ Despite recent changes to the Diagnostic and Statistical Manual of Mental Disorders-5 (DSM-V), which OCD removed from the anxiety section of the DSM and gave a chapter of its own, called Obsessive-Compulsive and Related Disorders, the core clinical features of OCD remain the same. ${ }^{3,4}$ Individuals with OCD may demonstrate a diversity of obsessions and compulsions. The YaleBrown Obsessive Compulsive Scale (Y-BOCS), most widely used by OCD researchers 
and clinicians, is designed to categorize the type of symptoms and assess the severity of OCD. ${ }^{5}$

OCD prevalence is estimated to be between $1.5 \%$ and $3 \%$ of the people worldwide, independent of ethnicity and cultural group studied. ${ }^{6}$ It is expected to become one of the top 10 leading causes of disability worldwide within the next 20 years. $^{7}$ The study conducted by Koran to evaluate the quality of life in OCD patients has shown moderate or severe interference with socializing, family relationships, self-esteem and the ability to study and work. ${ }^{8}$ These functional impairments have burdened a significant expense on the society. It was indicated that the disability associated with OCD was about $47 \%$, and the costs were estimated to be 10.6 billion dollars per annum in the US. ${ }^{9}$ Furthermore, studies ${ }^{10-12}$ manifest that suicidal tendencies in OCD patients have been underestimated, and the rate of suicidality (suicidal thoughts, attempts and completed suicide) is reported to be from $20 \%$ to $46 \%$ among OCD sufferers. ${ }^{13}$ Considering its severity, high prevalence and apparent social cost, OCD has to be studied more extensively; there is a pressing need for further research to gain an improved recognition of its etiology and treatment.

Many etiological studies on OCD confirm that OCD is familial and heritable. ${ }^{14-18}$ Family aggregation studies, the result from twin studies, functional neuroimaging, pharmacologic and molecular genetic studies have demonstrated convincing data for the genetic basis of OCD. ${ }^{19,20}$ Increasing progress in recent molecular biology techniques has led to an impressive interest to identify which genes are entailed in OCD etiology. Although a considerable segment of the genetic contribution to OCD remains unknown, genome-wide association studies and candidate gene studies provide convincing evidence for the importance of specific genes that may be involved in the expression of OCD. ${ }^{21}$ Moreover, several evidence are suggesting the impact of genetic factors on the response to drug treatment. Identification of biologic factors, which may be related to treatment response, could be extremely beneficial to gain appropriate clinical outcome. ${ }^{22}$ Based on the efficacy of selective serotonin reuptake inhibitors (SSRIs) in treating $\mathrm{OCD},{ }^{23}$ many genes with a role in the serotonergic system have been examined in genetic and pharmacogenetic studies. ${ }^{19,24}$

A large family of serotonin (5-hydroxytryptamine [5-HT]) receptors has been recognized, including $5 H T 2 A, H T R 1 B$ and 5-HT2C, and their associations with obsessive-compulsive symptoms and OCD treatment response have been studied extensively. ${ }^{19,24-26}$ Serotonin receptor $2 \mathrm{a}$ is a critical receptor in the serotonergic pathway, which was found to be associated with many different behavioral disorders such as $\mathrm{OCD},{ }^{27}$ eating disorders, ${ }^{28}$ schizophrenia, ${ }^{29}$ alcoholism,,${ }^{30}$ depression ${ }^{31}$ and suicidal behavior. ${ }^{32}$ The gene for the 5-HT2A receptor, HTR $2 A$, is located on chromosome 13q14-q21. ${ }^{33}$ It spans $20 \mathrm{~kb}$ and includes three exons containing $>200$ single-nucleotide polymorphisms (SNPs) along the gene. ${ }^{32}$ Two of the most remarkable SNPs of the HTR2A which were extensively studied in the OCD are rs6311 (1438G/A) and rs6313 (102C/T)..$^{19,27,33-38}$ However, studies testing the hypothesis that allelic variations of the HTR $2 A$ could be associated with the drug response in OCD are limited. ${ }^{39}$ Although the functional significance of these polymorphisms is not entirely understood, there are pieces of evidence that rs6311 modulates transcription factor binding and promoter methylation, affecting gene transcription. ${ }^{40}$

The current study aimed to investigate the presence of the association between the HTR $2 A$ polymorphism rs6311 and rs6313 and OCD and its clinical characteristics, as well as their role in OCD SSRI treatment responsiveness in Iranian population.

\section{Subjects and methods Subjects}

In this study, the subjects consisted of 293 patients with OCD recruited from Imam Hossain Hospital (Tehran, Iran) and 245 healthy controls. The diagnosis of OCD was established by a psychiatrist based on DSM-IV-TR criteria for OCD. Obsessive and compulsive types and severities were evaluated by an expert psychologist through the structured interview using Y-BOCS checklist and severity scale. Participants were aged between 18 and 65 years. Also, patients with total Y-BOCS severity $<9$, having OCD symptoms for $<1$ year, reporting a history of psychotic disorders, mental retardation, severe neurologic pathology and other DSM-IV-TR Axis 1 disorders except for depression and anxiety, as well as those being under the SSRI or antidepressant pharmacotherapy were excluded. All patients and controls were matched for sex and age. The demographic characteristics of the patients are summarized in Table 1. All participants gave written informed consent before they were included in the study. The study was approved by the Research Ethics Committee of Neuroscience Research Center, Shahid Beheshti University of Medical Sciences, Tehran, Iran (IR.SBMU.PHNS. REC.1396.69).

\section{Clinical data}

Sociodemographic data were gathered by an interview based on a questionnaire including name and address, age at assessment, sex, marital status, ethnicity, educational level, occupation, age of onset (if symptoms started before 18 years of age, the patients were classified as early onset; otherwise, they were endorsed late onset ${ }^{41}$ ), illness duration, history of substance 
Table I Sociodemographic characteristics of OCD patients $(\mathrm{N}=293)$

\begin{tabular}{|c|c|c|}
\hline & $\mathbf{n}$ & $\%$ \\
\hline \multicolumn{3}{|l|}{ Sex } \\
\hline Female & 204 & 69.6 \\
\hline Male & 89 & 30.3 \\
\hline \multicolumn{3}{|l|}{ Marital status } \\
\hline Single & 82 & 27.9 \\
\hline Married & 211 & 72.0 \\
\hline \multicolumn{3}{|l|}{ Level of education } \\
\hline School dropout & 87 & 29.6 \\
\hline Diploma & 133 & 45.3 \\
\hline Undergraduate & 59 & 20.1 \\
\hline Graduate & 12 & 4.0 \\
\hline Missing & 2 & 0.6 \\
\hline \multicolumn{3}{|l|}{ Occupation } \\
\hline Unemployed & 175 & 59.7 \\
\hline Employed & 117 & 39.9 \\
\hline Missing & I & 0.3 \\
\hline \multicolumn{3}{|l|}{ Age of onset } \\
\hline Early & 82 & 27.9 \\
\hline Late & 210 & 71.6 \\
\hline Missing & I & 0.3 \\
\hline \multicolumn{3}{|c|}{ Familial history of psychiatric disorders } \\
\hline Positive history & 233 & 79.5 \\
\hline \multirow[t]{2}{*}{ Negative history } & 60 & 20.4 \\
\hline & Mean & SD \\
\hline \multicolumn{3}{|l|}{ Age (years) } \\
\hline Age at assessment & 35.03 & 10.34 \\
\hline Age of symptoms onset & 24.73 & 10.77 \\
\hline \multicolumn{3}{|c|}{ Y-BOCS current severity } \\
\hline Obsession & 10.89 & 4.65 \\
\hline Compulsion & 9.40 & 5.66 \\
\hline Total score & 20 & 8.63 \\
\hline
\end{tabular}

Abbreviations: OCD, obsessive-compulsive disorder; Y-BOCS, Yale-Brown Obsessive Compulsive scale.

use and the familial history of any psychiatric disorders, specifically OCD. In order to clarify the symptom and estimate the severity, the Persian version of Y-BOCS validated by Rajezi Esfahani et al ${ }^{42}$ was applied. The Global Assessment of Functioning was evaluated by the psychiatrist according to DSM-V Global Assessment of Functioning scale.

\section{Study protocol}

In this study, pharmacotherapy was defined as 12 weeks of treatment with fluvoxamine (100-300 mg). No concomitant therapy was allowed during the entire treatment period, either pharmacologic or nonpharmacologic. Of 352 patients who had participated in the current study, 293 patients were eligible for examination of genetic association analyses and about 131 patients completed their pharmacotherapy and were included in the pharmacogenetic study (Figure 1).

According to the reduction in patients' Y-BOCS score at the beginning of the treatment compared to the score after 12 weeks of treatment with fluvoxamine, patients were divided into two groups: group A (responders) was composed of patients who exhibited $>25 \%$ reduction in Y-BOCS scores after treatment with fluvoxamine and group B (nonresponders) comprised patients who exhibited $<25 \%$ reduction in Y-BOCS scores. ${ }^{43,44} \mathrm{We}$ also included another group (refractory patients) consisting of patients who experienced various SSRI trials during their illness period, but did not respond to them adequately. ${ }^{45}$

\section{Genotyping}

Venous blood $(6 \mathrm{~mL})$ of OCD patients and healthy controls was drawn, and DNA extraction was done by salting out method from peripheral blood cells according to the method which has been previously described. ${ }^{46}$ After DNA extraction, polymerase chain reaction (PCR) amplification was performed using Taq DNA polymerase 2x Master Mix (Ampliqon, Odense, Denmark; www. ampliqon.com) with specific primers for rs6311 (forward: 5'-AACCAACTTATTTCCTACCA-3' and reverse: 5'-AAGCTGCAAGGTAGCAACAG-3') and rs6313 (forward: 5'-AGCTCAACTACGAACTCCCT-3' and 5'-GTAAGGAGAGACACGACGGT-3'). All the PCR reactions were done on a Bio-Rad T100 Thermal Cycler under the following program: $95^{\circ} \mathrm{C}$ for $7 \mathrm{~min}, 30$ cycles of three repeating steps (denaturation at $95^{\circ} \mathrm{C}$ for $45 \mathrm{~s}$, annealing at $58^{\circ} \mathrm{C}$ for $30 \mathrm{~s}$ and extension at $72^{\circ} \mathrm{C}$ for $30 \mathrm{~s}$ ) and one final extension cycle at $72^{\circ} \mathrm{C}$ for $5 \mathrm{~min}$. The PCR products were digested with MspI restriction enzyme (Cat No \#ER0541) for both SNPs and were subsequently loaded on 3\% agarose gel to distinguish between different variants. Genotypes were confirmed by Sanger sequencing.

\section{Statistical analysis}

Allelic and genotypic frequencies were subjected to SPSS (version 19.0; IBM Corporation, Armonk, NY, USA) to evaluate differences between the patient and control groups as well as the patients' stratified groups considering treatment responsiveness, sex and familial history of psychiatric disorders in different genetic models using chi-square statistics. The Hardy-Weinberg (H-W) equilibrium and haplotype analysis were also performed by means of chi-square test. After Bonferroni correction, statistical significance was assumed at the $P$-value $<0.008$.

\section{Results \\ SNP association analyses with OCD}

The genotype distribution was tested by chi statistics and found to be in $\mathrm{H}-\mathrm{W}$ equilibrium both in control subjects 


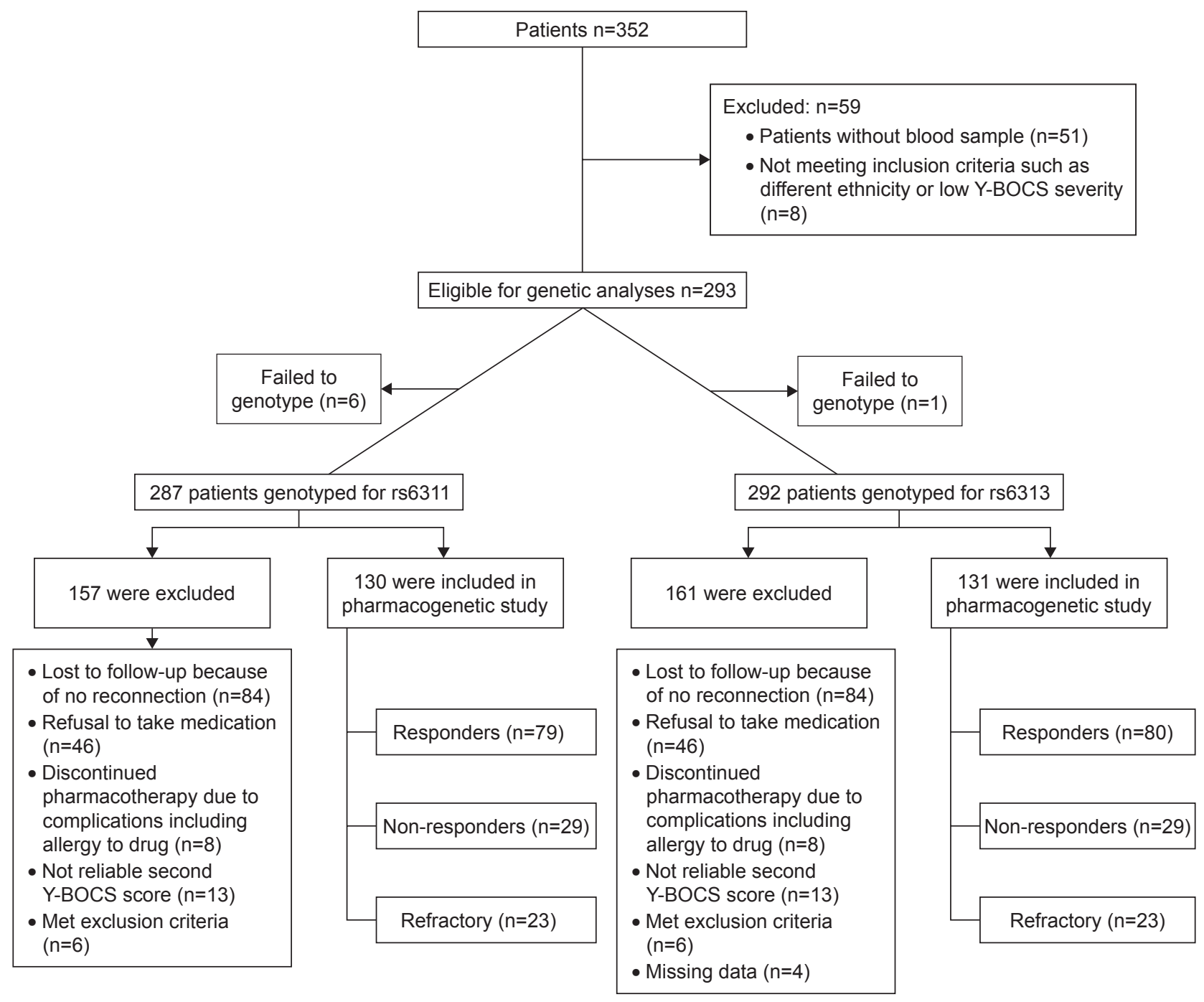

Figure I Flow diagram of the study progress.

Abbreviation: Y-BOCS, Yale-Brown Obsessive Compulsive Scale.

and patients $(P=0.49$ for rs6311 and $P=0.46$ for rs6313 in patients and $P=0.49$ for $\mathrm{rs} 6311$ and $P=0.89$ for rs6313 in controls).

Table 2 shows the results of association analyses of rs6311 and rs6313 with OCD based on different genetic models (co-dominance, dominant and recessive) considering sex. Results showed that none of the studied SNPs were associated with OCD in Iranian patients.

\section{SNP association analyses with familial form of OCD}

Results of genotypic association analyses with the family history of psychiatric disorders considering sex in different genetic models are summarized in Table 3 . When comparing the genotype distributions among patients according to the presence of family history of psychiatric disorders, association analysis of rs6311 showed no association with familial form of OCD. However, association analysis detected association of rs6313 with the presence of psychiatric disorder history under the recessive genetic model in female patients (Table 3).

\section{Haplotype association analysis}

To examine the association of HTR $2 a$ haplotypes with OCD, haplotype analysis of the investigated polymorphisms was conducted. Haplotypes were defined as H1 (GC), H2 (GT), $\mathrm{H} 3$ (AC) and H4 (AT). The results showed significant differences between the haplotype frequencies of OCD patients and controls (Table 4). OCD patients had less GC haplotype and more AC haplotype compared to controls.

\section{Pharmacogenetic analysis}

Results of single locus association studies with fluvoxamine treatment response in OCD patients considering sex are 
Table 2 Genotype distribution of the HTR2A polymorphisms in OCD patients and controls under different genetic models considering sex

\begin{tabular}{|c|c|c|c|c|c|c|c|}
\hline \multirow[t]{2}{*}{ rs63II } & \multicolumn{2}{|c|}{ Controls } & \multicolumn{2}{|c|}{ Patients } & \multirow[t]{2}{*}{$P$-value } & \multirow[t]{2}{*}{ OR } & \multirow[t]{2}{*}{ Lower-upper } \\
\hline & $\mathbf{n}$ & $\%$ & $\mathbf{n}$ & $\%$ & & & \\
\hline \multicolumn{8}{|c|}{ Co-dominance } \\
\hline \multicolumn{8}{|l|}{ Female } \\
\hline GG & 27 & 23.4 & 61 & 30.3 & 0.310 & & \\
\hline GA & 64 & 55.6 & 95 & 47.3 & & & \\
\hline $\mathrm{AA}$ & 24 & 22 & 45 & 23.4 & & & \\
\hline \multicolumn{8}{|l|}{ Male } \\
\hline GG & 24 & 19.5 & 26 & 30.0 & 0.065 & & \\
\hline GA & 68 & 55.2 & 34 & 39.6 & & & \\
\hline AA & 31 & 25.2 & 26 & 30.3 & & & \\
\hline \multicolumn{8}{|l|}{ Total } \\
\hline GG & 51 & 21.4 & 87 & 30.3 & 0.031 & & \\
\hline GA & 132 & 55.4 & 129 & 44.9 & & & \\
\hline $\mathrm{AA}$ & 55 & 23.3 & 71 & 24.7 & & & \\
\hline \multicolumn{8}{|c|}{ Dominance } \\
\hline \multicolumn{8}{|l|}{ Female } \\
\hline $\mathrm{GG}+\mathrm{GA}$ & 91 & 79.1 & 156 & 77.6 & 0.753 & 1.094 & $0.626-1.912$ \\
\hline $\mathrm{AA}$ & 24 & 20.8 & 45 & 22.3 & & & \\
\hline \multicolumn{8}{|l|}{ Male } \\
\hline GG+GA & 92 & 74.7 & 60 & 69.7 & 0.422 & 1.028 & $0.696-2.377$ \\
\hline $\mathrm{AA}$ & 31 & 25.2 & 26 & 30.23 & & & \\
\hline \multicolumn{8}{|l|}{ Total } \\
\hline $\mathrm{GG}+\mathrm{GA}$ & 183 & 76.8 & 216 & 75.2 & 0.662 & 1.094 & $0.73 \mathrm{I}-1.637$ \\
\hline $\mathrm{AA}$ & 55 & 23.1 & 71 & 24.7 & & & \\
\hline \multicolumn{8}{|l|}{ Recessive } \\
\hline \multicolumn{8}{|l|}{ Female } \\
\hline GG & 27 & 23.4 & 63 & 31.3 & 0.126 & 0.672 & $0.398-1.135$ \\
\hline $\mathrm{GA}+\mathrm{AA}$ & 88 & 76.5 & 138 & 68.6 & & & \\
\hline \multicolumn{8}{|l|}{ Male } \\
\hline GG & 24 & 19.5 & 26 & 30.2 & 0.074 & 0.559 & $0.295-1.062$ \\
\hline $\mathrm{GA}+\mathrm{AA}$ & 99 & 80.4 & 60 & 69.7 & & & \\
\hline \multicolumn{8}{|l|}{ Total } \\
\hline GG & 51 & 21.4 & 89 & 31.0 & 0.013 & 0.607 & $0.408-0.903$ \\
\hline $\mathrm{GA}+\mathrm{AA}$ & 187 & 78.5 & 198 & 68.9 & & & \\
\hline rs6313 & Con & & Pati & & $P$-value & OR & Lower-upper \\
\hline & $\mathbf{n}$ & $\%$ & $\mathbf{n}$ & $\%$ & & & \\
\hline Co-domina & & & & & & & \\
\hline Female & & & & & & & \\
\hline $\mathrm{CC}$ & 29 & 25 & 63 & 31.0 & 0.465 & & \\
\hline $\mathrm{CT}$ & 61 & 52.5 & 94 & 46.3 & & & \\
\hline TT & 26 & 22.4 & 46 & 22.6 & & & \\
\hline Male & & & & & & & \\
\hline CC & 34 & 27.4 & 27 & 30.3 & 0.204 & & \\
\hline CT & 64 & 51.6 & 36 & 40.4 & & & \\
\hline TT & 26 & 20.9 & 26 & 29.2 & & & \\
\hline Total & & & & & & & \\
\hline $\mathrm{CC}$ & 63 & 26.2 & 90 & 30.8 & 0.180 & & \\
\hline $\mathrm{CT}$ & 125 & 52.0 & 130 & 44.5 & & & \\
\hline $\mathrm{TT}$ & 52 & 21.6 & 72 & 24.6 & & & \\
\hline Dominance & & & & & & & \\
\hline Female & & & & & & & \\
\hline $\mathrm{CC}+\mathrm{CT}$ & 90 & 77.3 & 157 & 77.3 & 0.992 & 1.003 & $0.58 \mathrm{I}-1.733$ \\
\hline TT & 26 & 22.6 & 46 & 22.6 & & & \\
\hline & & & & & & & (Continued) \\
\hline
\end{tabular}


Table 2 (Continued)

\begin{tabular}{|c|c|c|c|c|c|c|c|}
\hline \multirow[t]{2}{*}{ rs6313 } & \multicolumn{2}{|c|}{ Controls } & \multicolumn{2}{|c|}{ Patients } & \multirow[t]{2}{*}{$P$-value } & \multirow[t]{2}{*}{ OR } & \multirow[t]{2}{*}{ Lower-upper } \\
\hline & $\mathbf{n}$ & $\%$ & $\mathbf{n}$ & $\%$ & & & \\
\hline \multicolumn{8}{|l|}{ Male } \\
\hline $\mathrm{CC}+\mathrm{CT}$ & 98 & 79.2 & 63 & 70.7 & 0.157 & $1.57 \mid$ & $0.838-2.947$ \\
\hline TT & 26 & 20.8 & 26 & 29.2 & & & \\
\hline \multicolumn{8}{|l|}{ Total } \\
\hline $\mathrm{CC}+\mathrm{CT}$ & 188 & 78.3 & 220 & 75.3 & 0.417 & 1.183 & $0.788-1.776$ \\
\hline TT & 52 & 21.6 & 72 & 24.6 & & & \\
\hline \multicolumn{8}{|l|}{ Recessive } \\
\hline \multicolumn{8}{|l|}{ Female } \\
\hline $\mathrm{CC}$ & 29 & 25.2 & 63 & 31.0 & 0.272 & 0.749 & $0.448-1.255$ \\
\hline $\mathrm{CT}+\mathrm{TT}$ & 87 & 74.7 & 140 & 68.9 & & & \\
\hline \multicolumn{8}{|l|}{ Male } \\
\hline $\mathrm{CC}$ & 34 & 27.2 & 27 & 30.3 & 0.616 & 0.858 & $0.47 \mid-1.563$ \\
\hline $\mathrm{CT}+\mathrm{TT}$ & 90 & 72.8 & 62 & 69.6 & & & \\
\hline \multicolumn{8}{|l|}{ Total } \\
\hline CC & 63 & 26.2 & 90 & 30.8 & 0.246 & 0.799 & $0.546-1.168$ \\
\hline $\mathrm{CT}+\mathrm{TT}$ & 177 & 73.7 & 202 & 69.6 & & & \\
\hline
\end{tabular}

Note: Statistical significance considered as $P$-value $<0.008$.

Abbreviations: $O C D$, obsessive-compulsive disorder; $O R$, odds ratio.

Table 3 Genotype distribution of the HTR2A polymorphisms in familial form of OCD under different genetic models considering sex

\begin{tabular}{|c|c|c|c|c|c|c|c|}
\hline \multirow[t]{3}{*}{ rs63II } & \multicolumn{4}{|c|}{ Family history } & \multirow[t]{3}{*}{$P$-value } & \multirow[t]{3}{*}{ OR } & \multirow[t]{3}{*}{ Lower-upper } \\
\hline & \multicolumn{2}{|c|}{ Negative } & \multicolumn{2}{|c|}{ Positive } & & & \\
\hline & $\bar{n}$ & $\%$ & $\bar{n}$ & $\%$ & & & \\
\hline \multicolumn{8}{|c|}{ Co-dominance } \\
\hline \multicolumn{8}{|c|}{ Female } \\
\hline GG & 23 & 46 & 39 & 26 & 0.040 & & \\
\hline GA & 17 & 34 & 76 & 50.6 & & & \\
\hline AA & 10 & 20 & 35 & 23.3 & & & \\
\hline \multicolumn{8}{|l|}{ Male } \\
\hline GG & I & 12.5 & 24 & 31.1 & 0.375 & & \\
\hline GA & 3 & 37.5 & 31 & 40.2 & & & \\
\hline AA & 4 & 50 & 22 & 28.5 & & & \\
\hline \multicolumn{8}{|l|}{ Total } \\
\hline GG & 24 & 40.3 & 63 & 27.7 & 0.144 & & \\
\hline GA & 20 & 35.0 & 107 & 47.1 & & & \\
\hline $\mathrm{AA}$ & 14 & 24.5 & 57 & 25.1 & & & \\
\hline \multicolumn{8}{|c|}{ Dominance } \\
\hline \multicolumn{8}{|c|}{ Female } \\
\hline $\mathrm{GG}+\mathrm{CT}$ & 40 & 80 & 115 & 76.6 & 0.391 & 1.217 & $0.553-2.68$ I \\
\hline AA & 10 & 20 & 35 & 23.3 & & & \\
\hline \multicolumn{8}{|l|}{ Male } \\
\hline $\mathrm{GG}+\mathrm{CT}$ & 4 & 50 & 55 & 76.6 & 0.195 & 0.400 & $0.092-1.742$ \\
\hline AA & 4 & 50 & 22 & 71.4 & & & \\
\hline \multicolumn{8}{|l|}{ Total } \\
\hline $\mathrm{GG}+\mathrm{CT}$ & 44 & 75.8 & 170 & 74.8 & 0.514 & 1.054 & $0.538-2.064$ \\
\hline AA & 14 & 24.1 & 57 & 25.1 & & & \\
\hline \multicolumn{8}{|l|}{ Recessive } \\
\hline \multicolumn{8}{|l|}{ Female } \\
\hline GG & 22 & 44 & 39 & 71.4 & 0.014 & 2.236 & I.148-4.357 \\
\hline $\mathrm{GA}+\mathrm{AA}$ & 28 & 56 & 111 & 28.5 & & & \\
\hline \multicolumn{8}{|l|}{ Male } \\
\hline GG & I & 12.5 & 24 & 31.1 & 0.254 & 0.315 & $0.037-2.708$ \\
\hline $\mathrm{GA}+\mathrm{AA}$ & 7 & 87.5 & 53 & 68.8 & & & \\
\hline \multicolumn{8}{|l|}{ Total } \\
\hline GG & 23 & 39.6 & 63 & 27.7 & 0.056 & 1.711 & $0.938-3.120$ \\
\hline $\mathrm{GA}+\mathrm{AA}$ & 35 & 60.3 & 164 & 72.2 & & & \\
\hline
\end{tabular}


Table 3 (Continued)

\begin{tabular}{|c|c|c|c|c|c|c|c|}
\hline \multirow[t]{3}{*}{ rs6313 } & \multicolumn{4}{|c|}{ Family history } & \multirow[t]{3}{*}{$P$-value } & \multirow[t]{3}{*}{ OR } & \multirow[t]{3}{*}{ Lower-upper } \\
\hline & \multicolumn{2}{|c|}{ Negative } & \multicolumn{2}{|c|}{ Positive } & & & \\
\hline & n & $\%$ & $n$ & $\%$ & & & \\
\hline \multicolumn{8}{|c|}{ Co-dominance } \\
\hline \multicolumn{8}{|c|}{ Female } \\
\hline $\mathrm{CC}$ & 24 & 47.0 & 39 & 25.8 & 0.017 & & \\
\hline $\mathrm{CT}$ & 17 & 33.2 & 76 & 50.3 & & & \\
\hline $\mathrm{TT}$ & 10 & 19.6 & 36 & 23.8 & & & \\
\hline \multicolumn{8}{|l|}{ Male } \\
\hline CC & I & II.I & 25 & 31.6 & 0.380 & & \\
\hline $\mathrm{CT}$ & 4 & 44.4 & 32 & 40.5 & & & \\
\hline TT & 4 & 44.4 & 22 & 27.8 & & & \\
\hline \multicolumn{8}{|l|}{ Total } \\
\hline CC & 25 & 41.6 & 64 & 27.8 & 0.102 & & \\
\hline $\mathrm{CT}$ & 21 & 35 & 108 & 46.9 & & & \\
\hline TT & 14 & 23.3 & 58 & 25.2 & & & \\
\hline \multicolumn{8}{|c|}{ Dominance } \\
\hline \multicolumn{8}{|c|}{ Female } \\
\hline $\mathrm{CC}+\mathrm{CT}$ & 41 & 80.3 & 115 & 76.1 & 0.533 & 1.282 & $0.585-2.817$ \\
\hline TT & 10 & 19.6 & 36 & 23.8 & & & \\
\hline \multicolumn{8}{|l|}{ Male } \\
\hline $\mathrm{CC}+\mathrm{CT}$ & 5 & 55.5 & 57 & 72.1 & 0.301 & 0.482 & $0.119-1.964$ \\
\hline $\mathrm{TT}$ & 4 & 44.4 & 22 & 27.8 & & & \\
\hline \multicolumn{8}{|l|}{ Total } \\
\hline $\mathrm{CC}+\mathrm{CT}$ & 46 & 76.6 & 172 & 74.7 & 0.764 & 1.108 & $0.568-2.161$ \\
\hline TT & 14 & 23.3 & 58 & 25.2 & & & \\
\hline \multicolumn{8}{|l|}{ Recessive } \\
\hline \multicolumn{8}{|l|}{ Female } \\
\hline $\mathrm{CC}$ & 24 & 47.0 & 39 & 25.8 & $0.005^{*}$ & 2.553 & $1.320-4.937$ \\
\hline $\mathrm{CT}+\mathrm{TT}$ & 27 & 52.9 & 112 & 74.1 & & & \\
\hline \multicolumn{8}{|l|}{ Male } \\
\hline $\mathrm{CC}$ & I & II.I & 25 & 31.6 & 0.201 & 0.270 & $0.032-2.277$ \\
\hline $\mathrm{CT}+\mathrm{TT}$ & 8 & 88.8 & 54 & 68.3 & & & \\
\hline \multicolumn{8}{|l|}{ Total } \\
\hline CC & 25 & 41.6 & 64 & 27.8 & 0.038 & 1.83 & $1.028-3.338$ \\
\hline $\mathrm{CT}+\mathrm{TT}$ & 35 & 58.3 & 166 & 72.1 & & & \\
\hline
\end{tabular}

Note: ${ }^{* P}$-value $<0.008$.

Abbreviations: $\mathrm{OCD}$, obsessive-compulsive disorder; OR, odds ratio.

summarized in Table 5. Comparing the genotypic distribution in different treatment response groups (responders, nonresponders and refractory patients) showed no significant differences for both the SNPs studied (Table 5). However, two HTR $2 A$ haplotypes were associated with fluvoxamine

Table 4 Haplotype association analysis of HTR2A polymorphisms rs63II and rs63। 3 with OCD

\begin{tabular}{|c|c|c|c|c|c|c|c|}
\hline \multirow[t]{2}{*}{ Haplotype } & \multirow[t]{2}{*}{ rs63II } & \multirow[t]{2}{*}{ rs6313 } & \multicolumn{2}{|c|}{ Controls } & \multicolumn{2}{|c|}{ Patients } & \multirow[t]{2}{*}{$P$-value } \\
\hline & & & $\mathbf{n}$ & $\%$ & $\mathbf{n}$ & $\%$ & \\
\hline $\mathrm{HI}$ & G & C & 467 & 40.9 & 295 & 31.2 & $0.047 *$ \\
\hline $\mathrm{H} 2$ & G & $\mathrm{T}$ & 135 & 11.8 & 167 & 17.6 & 0.117 \\
\hline $\mathrm{H} 3$ & $A$ & C & 141 & 12.3 & 197 & 20.8 & $0.029 *$ \\
\hline $\mathrm{H} 4$ & A & $\mathrm{T}$ & 397 & 34.8 & 285 & 30.1 & 0.336 \\
\hline
\end{tabular}

treatment response in OCD (Table 6). The responder group had more AC and less AT haplotypes compared to the nonresponder group.

\section{Discussion}

In this study, we hypothesized that $H T R 2 A$ polymorphisms, rs6311 and rs6313, are associated with OCD and fluvoxamine treatment response in OCD patients. Results showed that each single locus was not associated with OCD, but their haplotypes were associated with OCD and its treatment response. Moreover, rs6313 was associated with the familial form of OCD in females.

To our knowledge, this is the first study addressing the genetic association of rs6311 and rs6313 with OCD in Iranian population and evaluating their associations with 
Table 5 Genetic association analyses of the HTR2A polymorphisms with OCD treatment response under different genetic models considering sex

\begin{tabular}{|c|c|c|c|c|c|c|c|}
\hline \multirow[t]{2}{*}{ rs63II } & \multicolumn{2}{|c|}{ Responders } & \multicolumn{2}{|c|}{ Non-responders } & \multicolumn{2}{|c|}{ Refractory } & \multirow[t]{2}{*}{$P$-value } \\
\hline & $\mathbf{n}$ & $\%$ & $\mathbf{n}$ & $\%$ & $\mathbf{n}$ & $\%$ & \\
\hline \multicolumn{8}{|c|}{ Co-dominance } \\
\hline \multicolumn{8}{|c|}{ Female } \\
\hline GG & 11 & 21.5 & 5 & 25 & 3 & 20 & $0.28 \mathrm{I}$ \\
\hline GA & 29 & 56.8 & 6 & 30 & 8 & 53.3 & \\
\hline AA & 11 & 21.5 & 9 & 45 & 4 & 26.6 & \\
\hline \multicolumn{8}{|l|}{ Male } \\
\hline GG & 7 & 25 & 2 & 22.2 & 1 & 12.5 & 0.856 \\
\hline GA & 11 & 39.8 & 5 & 55.5 & 4 & 50 & \\
\hline $\mathrm{AA}$ & 10 & 35.7 & 2 & 22.2 & 3 & 37.5 & \\
\hline \multicolumn{8}{|l|}{ Total } \\
\hline GG & 18 & 22.78 & 7 & 24.1 & 4 & 17.3 & 0.730 \\
\hline GA & 40 & 50.6 & 11 & 37.9 & 12 & 52.1 & \\
\hline AA & 21 & 26.5 & 11 & 37.9 & 7 & 30.4 & \\
\hline \multicolumn{8}{|c|}{ Dominance } \\
\hline \multicolumn{8}{|l|}{ Female } \\
\hline GG+GA & 40 & 50.6 & II & 55 & II & 73.3 & 0.140 \\
\hline AA & 11 & 13.9 & 9 & 45 & 4 & 26.6 & \\
\hline \multicolumn{8}{|l|}{ Male } \\
\hline $\mathrm{GG}+\mathrm{GA}$ & 18 & 64.2 & 7 & 77.7 & 5 & 62.5 & 0.728 \\
\hline $\mathrm{AA}$ & 10 & 35.7 & 2 & 22.2 & 3 & 37.5 & \\
\hline \multicolumn{8}{|l|}{ Total } \\
\hline $\mathrm{GG}+\mathrm{GA}$ & 58 & 73.4 & 18 & 62.0 & 16 & 69.5 & 0.519 \\
\hline $\mathrm{AA}$ & 21 & 26.5 & II & 37.9 & 7 & 30.4 & \\
\hline \multicolumn{8}{|l|}{ Recessive } \\
\hline \multicolumn{8}{|l|}{ Female } \\
\hline GG & 11 & 21.5 & 5 & 55 & 3 & 20 & 0.930 \\
\hline $\mathrm{GA}+\mathrm{AA}$ & 40 & 78.4 & 15 & 45 & 12 & 80 & \\
\hline \multicolumn{8}{|l|}{ Male } \\
\hline GG & 7 & 25 & 2 & 77.7 & 1 & 12.5 & 0.755 \\
\hline $\mathrm{GA}+\mathrm{AA}$ & 21 & 75 & 7 & 22.2 & 7 & 87.5 & \\
\hline \multicolumn{8}{|l|}{ Total } \\
\hline GG & 18 & 22.7 & 7 & 24.1 & 4 & 17.3 & 0.824 \\
\hline $\mathrm{GA}+\mathrm{AA}$ & 61 & 77.2 & 22 & 75.8 & 19 & 82.6 & \\
\hline rs6313 & Res & & No & & Ref & & $P$-value \\
\hline & $\mathrm{n}$ & $\%$ & $\mathrm{n}$ & $\%$ & $\mathrm{n}$ & $\%$ & \\
\hline Co-domin & & & & & & & \\
\hline Female & & & & & & & \\
\hline $\mathrm{CC}$ & 13 & 25 & 6 & 30 & 3 & 20 & 0.111 \\
\hline $\mathrm{CT}$ & 29 & 55.7 & 5 & 25 & 9 & 60 & \\
\hline TT & 10 & 19.2 & 9 & 45 & 3 & 20 & \\
\hline Male & & & & & & & \\
\hline CC & 7 & 25 & 2 & 22.2 & 1 & 12.5 & 0.904 \\
\hline $\mathrm{CT}$ & 12 & 42.8 & 5 & 55.5 & 4 & 50 & \\
\hline TT & 10 & 32.1 & 2 & 22.2 & 3 & 37.5 & \\
\hline Total & & & & & & & \\
\hline CC & 20 & 25 & 8 & 27.5 & 4 & 17.3 & 0.436 \\
\hline $\mathrm{CT}$ & 41 & 51.2 & 10 & 34.4 & 13 & 56.5 & \\
\hline $\mathrm{TT}$ & 19 & 23.7 & 11 & 37.9 & 6 & 26.0 & \\
\hline Dominanc & & & & & & & \\
\hline Female & & & & & & & \\
\hline $\mathrm{CC}+\mathrm{CT}$ & 42 & 80.7 & II & 55 & 12 & 80 & 0.069 \\
\hline TT & 10 & 19.2 & 9 & 45 & 3 & 20 & \\
\hline Male & & & & & & & \\
\hline $\mathrm{CC}+\mathrm{CT}$ & 19 & 67.8 & 7 & 77.7 & 5 & 62.5 & 0.780 \\
\hline $\mathrm{TT}$ & 9 & 32.1 & 2 & 22.2 & 3 & 37.5 & \\
\hline
\end{tabular}


Table 5 (Continued)

\begin{tabular}{|c|c|c|c|c|c|c|c|}
\hline \multirow[t]{2}{*}{ rs6313 } & \multicolumn{2}{|c|}{ Responders } & \multicolumn{2}{|c|}{ Non-responders } & \multicolumn{2}{|c|}{ Refractory } & \multirow[t]{2}{*}{$P$-value } \\
\hline & $\mathbf{n}$ & $\%$ & $\mathbf{n}$ & $\%$ & $\mathbf{n}$ & $\%$ & \\
\hline \multicolumn{8}{|l|}{ Total } \\
\hline $\mathrm{CC}+\mathrm{CT}$ & 61 & 76.2 & 18 & 62.0 & 17 & 73.9 & 0.337 \\
\hline TT & 19 & 23.7 & 11 & 37.9 & 6 & 26.0 & \\
\hline \multicolumn{8}{|l|}{ Recessive } \\
\hline \multicolumn{8}{|l|}{ Female } \\
\hline $\mathrm{CC}$ & 13 & 25 & 6 & 30 & 3 & 20 & 0.795 \\
\hline $\mathrm{CT}+\mathrm{TT}$ & 39 & 75 & 14 & 70 & 12 & 80 & \\
\hline \multicolumn{8}{|l|}{ Male } \\
\hline $\mathrm{CC}$ & 7 & 25 & 2 & 22.2 & I & 12.5 & 0.755 \\
\hline $\mathrm{CT}+\mathrm{TT}$ & 21 & 75 & 7 & 77.7 & 7 & 87.5 & \\
\hline \multicolumn{8}{|l|}{ Total } \\
\hline $\mathrm{CC}$ & 20 & 25 & 8 & 27.5 & 4 & 17.3 & 0.674 \\
\hline $\mathrm{CT}+\mathrm{TT}$ & 60 & 75 & 21 & 72.4 & 19 & 82.6 & \\
\hline
\end{tabular}

Note: Statistical significance considered as $P$-value $<0.008$

Abbreviation: OCD, obsessive-compulsive disorder.

OCD treatment response. However, their associations with OCD were studied in other populations.

A meta-analysis by Taylor showed that the A allele of rs6311 or the linked $\mathrm{T}$ allele of rs6313 was significantly associated with OCD. ${ }^{38}$ This result was confirmed in the author's subsequent comprehensive meta-analysis in 2016 with a larger dataset. ${ }^{47}$ Although many studies have reported this association, ${ }^{19,34}$ there are inconsistent reports which have not focused on the association of $H T R 2 A$ variants with OCD..$^{27,35,36}$ The findings of Nicolini et al showed the lack of significant association between rs6313 and OCD. ${ }^{36}$ In a cross-sectional study, Meira-Lima et al examined the allelic and genotypic frequencies of the rs6313 variants in 79 OCD patients and 202 control subjects and observed no significant difference between the patients and controls. ${ }^{27}$ Our evaluations in the Iranian population also showed no association of rs6311 or rs6313 with OCD at the single locus. However, the patterns of their haplotypes were significantly different between OCD cases and control subjects.

There are limited studies evaluating the genetic association in the stratified patients' groups specifically considering sex or familial form of OCD. Enoch et $\mathrm{al}^{48}$ indicated that the rs6311 A allele was more abundant in females with OCD compared to control females. Results from a study conducted by Denys et al accounted that GG genotype of rs6311 was more frequent in patients with a family history of psychiatric disorders. ${ }^{34}$ We could not detect the association of rs6311 with a positive family history of OCD, but we found the association of rs 6313 with the familial form of OCD in females. We previously detected the association of the functional serotonin transporter haplotype with the familial form of OCD in Iranian patients. ${ }^{46}$ Evidence for different genetic susceptibilities of the familial form of OCD compared to the non-familial form suggests different pathogenesis, which needs further investigation for a detailed elucidation.

The results of our pharmacogenetic study showed an association of $H T R 2 A$ haplotypes with fluvoxamine treatment response in the OCD patients. Pharmacogenetic evaluations of HTR $2 A$ polymorphisms with SSRI treatment response are limited with inconsistent results. Zhang et al studied 113 OCD nuclear families which underwent SRI pharmacotherapy for 8 weeks, and they assessed the association of six genes including HTR2A, 5-HTT, DRD2, DRD4, COMT and $M A O A$. Results showed no association between the six genes and different drug response groups, except for HTR $2 A$ rs6311. ${ }^{49}$ However, in another study examining 58 OCD

Table 6 Haplotype association analysis of HTR2A polymorphisms rs63II and rs63। 3 with OCD treatment response

\begin{tabular}{|c|c|c|c|c|c|c|c|c|c|}
\hline \multirow[t]{2}{*}{ Haplotype } & \multirow[t]{2}{*}{ rs63 I I } & \multirow[t]{2}{*}{ rs63 I3 } & \multicolumn{2}{|c|}{ Responders } & \multicolumn{2}{|c|}{ Non-responders } & \multicolumn{2}{|c|}{ Refractory } & \multirow[t]{2}{*}{ P-value } \\
\hline & & & n & $\%$ & n & $\%$ & n & $\%$ & \\
\hline $\mathrm{HI}$ & $\mathrm{G}$ & $C$ & 112 & 35.6 & 39 & 37.1 & 25 & 27.1 & 0.072 \\
\hline $\mathrm{H} 2$ & $\mathrm{G}$ & $\mathrm{T}$ & 38 & 12.1 & II & 10.4 & 15 & 16.3 & 0.185 \\
\hline $\mathrm{H} 3$ & $A$ & $\mathrm{C}$ & 46 & 14.6 & 0 & 0 & 17 & 18.4 & $0.000 I^{*}$ \\
\hline $\mathrm{H} 4$ & $A$ & $\mathrm{~T}$ & 118 & 37.5 & 55 & 52.3 & 35 & 38.0 & $0.004^{*}$ \\
\hline
\end{tabular}

Note: ${ }^{*} P$-value $<0.05$.

Abbreviation: $O C D$, obsessive-compulsive disorder. 
patients, no association between rs6311 and rs6313 with treatment response was reported.$^{50}$ Miguita et al also could not detect an influence of the rs6313 and 516C/T polymorphisms on treatment response in their sample of 41 OCD patients. ${ }^{51}$ Nonetheless, sample sizes in these studies are small, and using different SRIs is a confounding factor which may lead to inconsistencies in the results. Further studies in larger and more homogeneous clinical samples would help to have more explicit evidence related to the effects of these two SNPs on OCD treatment response.

This is the first study evaluating the role of HTR $2 A$ in OCD susceptibility and treatment response. The other strength of this study was prescribing a single drug, fluvoxamine, which avoided having confounding factors related to the different SSRI treatments. However, similar to any other research, we faced some limitations in this study. The most important limitation of this study is the sample size, so further replication studies with larger sample sizes are needed to confirm these preliminary findings. The other limitation is that we have not considered the environmental factors which may affect (positively or negatively) the treatment response.

\section{Conclusion}

We have found that the HTR $2 A$ haplotype is associated with OCD in Iranian population and with fluvoxamine treatment response in OCD patients. Association of rs6313 with the familial form of OCD in females suggests conducting further studies on the genetic background of the familial and non-familial forms of OCD, which may shed light on its pathogenesis and more specific treatment strategies.

\section{Acknowledgments}

This research was supported by grants from the Iran National Science Foundation and Neuroscience Research Center, Shahid Beheshti University of Medical Sciences, Iran. We would like to thank patients and their families for participating in this study and Imam Hossain Hospital staff for their cooperation with our research team. We also gratefully acknowledge NRC and Tarbiat Modarres graduate students and staff for participating in this study as our control group.

\section{Disclosure}

The authors report no conflicts of interest in this work.

\section{References}

1. Weil R, Feist A, Moritz S, Wittekind CE. Approaching contaminationrelated stimuli with an implicit approach-avoidance task: can it reduce OCD symptoms? An online pilot study. J Behav Ther Exp Psychiatry. 2017;57:180-188.
2. Alonso P, Menchón JM, Mataix-Cols D, et al. Perceived parental rearing style in obsessive-compulsive disorder: relation to symptom dimensions. Psychiatry Res. 2004;127(3):267-278.

3. Leckman JF, Denys D, Simpson HB, et al. Obsessive-compulsive disorder: a review of the diagnostic criteria and possible subtypes and dimensional specifiers for DSM-V. Depress Anxiety. 2010;27(6): 507-527.

4. Goodman WK, Grice DE, Lapidus KA, Coffey BJ. Obsessive-compulsive disorder. Psychiatr Clin. 2014;37(3):257-267.

5. Goodman WK, Price LH, Rasmussen SA, et al. The Yale-Brown obsessive compulsive scale: I. development, use, and reliability. Arch Gen Psychiatry. 1989;46(11):1006-1011.

6. Ahmari SE. Using mice to model obsessive compulsive disorder: from genes to circuits. Neuroscience. 2016;321:121-137.

7. Bloch MH, McGuire J, Landeros-Weisenberger A, Leckman JF, Pittenger C. Meta-analysis of the dose-response relationship of SSRI in obsessive-compulsive disorder. Mol Psychiatry. 2010;15(8):850-855.

8. Koran LM. Quality of life in obsessive-compulsive disorder. Psychiatr Clin North Am. 2000;23(3):509-517.

9. Eaton WW, Martins SS, Nestadt G, Bienvenu OJ, Clarke D, Alexandre P. The burden of mental disorders. Epidemiol Rev. 2008;30(1):1-14.

10. Fawzy N, Hashim H. Prevalence and risk factors of suicide among patients with obsessive-compulsive disorder. Middle East Curr Psychiatry. 2011;18(1):18-22.

11. Torres AR, de Abreu Ramos-Cerqueira AT, Torresan RC, de Souza Domingues M, Hercos ACR, Guimarães ABC. Prevalence and associated factors for suicidal ideation and behaviors in obsessive-compulsive disorder. CNS Spectr. 2007;12(10):771-778.

12. Kamath P, Reddy Y, Kandavel T. Suicidal behavior in obsessivecompulsive disorder. J Clin Psychiatry. 2007;68(11):1741-1750.

13. Brakoulias V, Starcevic V, Belloch A, et al. Comorbidity, age of onset and suicidality in obsessive-compulsive disorder (OCD): an international collaboration. Compr Psychiatry. 2017;76:79-86.

14. Sampaio AS, Lins RMP, Daltro-Oliveira R, et al. Genetic association studies in obsessive-compulsive disorder. Archives of Clinical Psychiatry (São Paulo). 2013;40(5):177-190.

15. van Grootheest DS, Cath DC, Beekman AT, Boomsma DI. Twin studies on obsessive-compulsive disorder: a review. Twin Res Hum Genet. 2005;8(5):450-458.

16. Nestadt G, Samuels J, Riddle M, et al. A family study of obsessivecompulsive disorder. Arch Gen Psychiatry. 2000;57(4):358-363.

17. Pauls DL. The genetics of obsessive-compulsive disorder: a review. Dialogues Clin Neurosci. 2010;12(2):149.

18. Grados MA, Walkup J, Walford S. Genetics of obsessive-compulsive disorders: new findings and challenges. Brain Dev. 2003;25:S55-S61.

19. Sinopoli V, Burton CL, Kronenberg S, Arnold PD. A review of the role of serotonin system genes in obsessive-compulsive disorder. Neurosci Biobehav Rev. 2017;80:372-381.

20. Rauch SL. Neuroimaging research and the neurobiology of obsessivecompulsive disorder: where do we go from here? Biol Psychiatry. 2000; 47(3):168-170.

21. Browne HA, Gair SL, Scharf JM, Grice DE. Genetics of obsessivecompulsive disorder and related disorders. Psychiatr Clin North Am. 2014;37(3):319-335.

22. Brandl EJ, Müller DJ, Richter MA. Pharmacogenetics of obsessivecompulsive disorders. Pharmacogenomics. 2012;13(1):71-81.

23. Goodman WK, Price LH, Delgado PL, et al. Specificity of serotonin reuptake inhibitors in the treatment of obsessive-compulsive disorder: comparison of fluvoxamine and desipramine. Arch Gen Psychiatry. 1990; 47(6):577-585.

24. Brandl EJ, Müller DJ, Richter MA. Pharmacogenetics of obsessivecompulsive disorders. Pharmacogenomics. 2012;13(1):71-81.

25. Saudou F, Hen R. 5-Hydroxytryptamine receptor subtypes in vertebrates and invertebrates. Neurochem Int. 1994;25(6):503-532.

26. Mundo E, Richter MA, Sam F, Macciardi F, Kennedy JL. Is the 5-HT1D $\beta$ receptor gene implicated in the pathogenesis of obsessivecompulsive disorder? Am J Psychiatry. 2000;157(7):1160-1161. 
27. Meira-Lima I, Shavitt R, Miguita K, Ikenaga E, Miguel E, Vallada H. Association analysis of the catechol-o-methyltransferase (COMT), serotonin transporter (5-HTT) and serotonin 2A receptor (5HT2A) gene polymorphisms with obsessive-compulsive disorder. Genes Brain Behav. 2004;3(2):75-79.

28. Brewerton TD. Toward a unified theory of serotonin dysregulation in eating and related disorders. Psychoneuroendocrinology. 1995; 20(6):561-590.

29. Sun L, Xu P, Zhou Y-G, Zuo S-R, Liu Y-P. Meta-analysis of polymorphism rs6311 and rs6313 in the 5-HT 2A R gene and schizophrenia. Nord J Psychiatry. 2017;71(1):1-11.

30. Wrzosek M, Łukaszkiewicz J, Wrzosek M, et al. Association of polymorphisms in HTR2A, HTR1A and TPH2 genes with suicide attempts in alcohol dependence: a preliminary report. Psychiatry Res. 2011; 190(1):149-151.

31. Khait VD, Yung-yu H, Zalsman G, et al. Association of serotonin 5-HT2A receptor binding and the T102C polymorphism in depressed and healthy Caucasian subjects. Neuropsychopharmacology. 2005; 30(1): 166 .

32. González-Castro TB, Tovilla-Zárate C, Juárez-Rojop I, et al. Association of the 5HTR2A gene with suicidal behavior: case-control study and updated meta-analysis. BMC Psychiatry. 2013;13(1):25.

33. Xu H, Guan J, Yi H, Yin S. A systematic review and meta-analysis of the association between serotonergic gene polymorphisms and obstructive sleep apnea syndrome. PLoS One. 2014;9(1):e86460.

34. Denys D, Van Nieuwerburgh F, Deforce D, Westenberg HG. Association between serotonergic candidate genes and specific phenotypes of obsessive compulsive disorder. J Affect Disord. 2006;91(1):39-44.

35. Frisch A, Michaelovsky E, Rockah R, et al. Association between obsessive-compulsive disorder and polymorphisms of genes encoding components of the serotonergic and dopaminergic pathways. Eur Neuropsychopharmacol. 2000;10(3):205-209.

36. Nicolini H, Cruz C, Camarena B, et al. DRD2, DRD3 and 5HT2A receptor genes polymorphisms in obsessive-compulsive disorder. Mol Psychiatry. 1996;1(6):461-465.

37. Liu W, Zhao N, Xiong J, Shi M, Hu J. Association analysis of serotonin and catecholamine system candidate genes in obsessive-compulsive disorder in the Chinese population. Psychiatry Res. 2011;188(1): 170-172.

38. Taylor S. Molecular genetics of obsessive-compulsive disorder: a comprehensive meta-analysis of genetic association studies. Mol Psychiatry. 2013;18(7):799-805.

39. Serretti A, Benedetti F, Zanardi R, Smeraldi E. The influence of Serotonin Transporter Promoter Polymorphism (SERTPR) and other polymorphisms of the serotonin pathway on the efficacy of antidepressant treatments. Prog Neuropsychopharmacol Biol Psychiatry. 2005; 29(6):1074-1084.
40. Falkenberg VR, Gurbaxani BM, Unger ER, Rajeevan MS. Functional genomics of serotonin receptor 2A (HTR2A): interaction of polymorphism, methylation, expression and disease association. Neuromolecular Med. 2011;13(1):66-76

41. Samuels JF, Riddle MA, Greenberg BD, et al. The OCD collaborative genetics study: methods and sample description. Am J Med Genet B Neuropsychiatr Genet. 2006;141(3):201-207.

42. RajeziEsfahani S, Motaghipour Y,Kamkari K, Zahiredin A, Janbozorgi M. Reliability and Validity of the Persian version of the Yale-Brown Obsessive-Compulsive scale (Y-BOCS). Iran J Psychiatry. 2012;17(4): 297-303.

43. Corregiari FM, Bernik M, Cordeiro Q, Vallada H. Endophenotypes and serotonergic polymorphisms associated with treatment response in obsessive-compulsive disorder. Clinics (Sao Paulo). 2012;67(4): $335-340$.

44. Bloch M, Landeros-Weisenberger A, Kelmendi B, Coric V, Bracken M, Leckman J. A systematic review: antipsychotic augmentation with treatment refractory obsessive-compulsive disorder. Mol Psychiatry. 2006;11(7):622-632.

45. Pallanti S, Quercioli L. Treatment-refractory obsessive-compulsive disorder: methodological issues, operational definitions and therapeutic lines. Prog Neuropsychopharmacol Biol Psychiatry. 2006;30(3): 400-412.

46. Rashidi FS, Ahmadipour E, Shiravand S, Ahmadiani A, Asadi S, Shams J. Association of the functional serotonin transporter haplotype with familial form of obsessive compulsive disorder in Iranian patients. Int J Psychiatry Clin Pract. 2017:1-7.

47. Taylor S. Disorder-specific genetic factors in obsessive-compulsive disorder: a comprehensive meta-analysis. Am JMed Genet B Neuropsychiatr Genet. 2016;171(3):325-332.

48. Enoch M-A, Greenberg BD, Murphy DL, Goldman D. Sexually dimorphic relationship of a 5-HT2A promoter polymorphism with obsessivecompulsive disorder. Biol Psychiatry. 2001;49(4):385-388.

49. Zhang L, Liu X-H, Li T, Yang Y, Hu X, Collier D. [Molecular pharmacogenetic studies of drug responses to obsessive-compulsive disorder and six functional genes]. Zhonghua Yi Xue Yi Chuan Xue Za Zhi. 2004; 21(5):479-481. Chinese.

50. Tot Ş, Erdal ME, Yazıcı K, Yazıcı AE, Metin Ö. T102C and -1438 G/A polymorphisms of the 5-HT2A receptor gene in Turkish patients with obsessive-compulsive disorder. Eur Psychiatry. 2003;18(5): 249-254.

51. Miguita K, Cordeiro Q, Shavitt RG, Miguel EC, Vallada H. Association study between genetic monoaminergic polymorphisms and OCD response to clomipramine treatment. Arq Neuropsiquiatr. 2011; 69(2B):283-287.
Neuropsychiatric Disease and Treatment

\section{Publish your work in this journal}

Neuropsychiatric Disease and Treatment is an international, peerreviewed journal of clinical therapeutics and pharmacology focusing on concise rapid reporting of clinical or pre-clinical studies on a range of neuropsychiatric and neurological disorders. This journa is indexed on PubMed Central, the 'PsycINFO' database and CAS,

\section{Dovepress}

and is the official journal of The International Neuropsychiatric Association (INA). The manuscript management system is completely online and includes a very quick and fair peer-review system, which is all easy to use. Visit http://www.dovepress.com/testimonials.php to read real quotes from published authors. 